\title{
Modelos de programação inteira mista para o planejamento do corte unidimensional de tubos metálicos na indústria aeronáutica agrícola
}

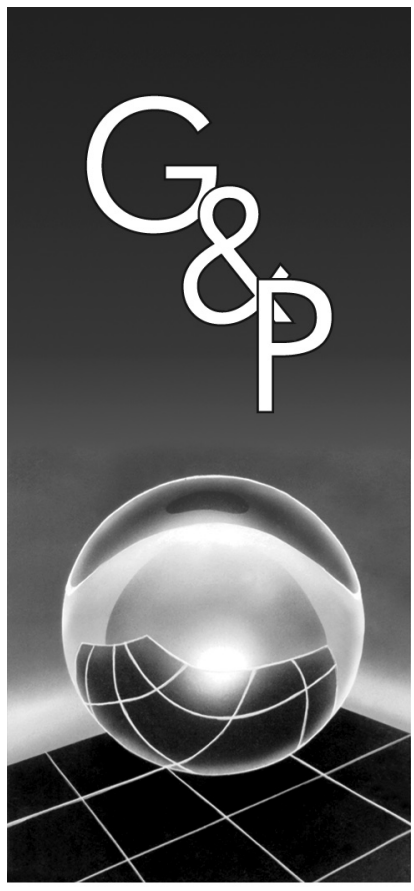

Alexander Abuabara

Reinaldo Morabito

\section{Resumo}

Este trabalho busca otimizar o planejamento do processo de corte unidimensional de tubos estruturais metálicos utilizados na fabricação de aeronaves leves agrícolas. Dois modelos de programação linear inteira mista são apresentados com o objetivo de minimizar as perdas do material cortado e considerando a possibilidade de gerar sobras com tamanhos suficientes para reaproveitamento (retalhos). Os modelos são resolvidos por meio de uma linguagem de modelagem usando um software de otimização. Para a validação dos modelos, dois experimentos computacionais foram realizados com dados reais de uma carteira de pedidos de uma aeronave leve voltada para o segmento do mercado agrícola, o Ipanema, produzido pela empresa brasileira Neiva/Embraer. As soluções dos modelos são comparadas com as soluções de uma heurística residual de arredondamento guloso da literatura e também com as soluções utilizadas pela empresa. Os resultados mostram que os modelos são úteis para apoiar as decisões envolvidas no planejamento deste processo de corte.

Palavras-chave: Planejamento de corte de tubos. Programação linear inteira mista. Indústria aeronáutica. Problemas de corte. Empacotamento unidimensional.

\section{Introdução}

O problema de corte de estoque (cutting stock problem) consiste em determinar a "melhor" forma de cortar unidades de material (objetos em estoque), produzindo um conjunto de unidades menores (itens demandados), com dimensões específicas, que são, em geral, encomendadas por meio de uma carteira de pedidos de clientes. Dependendo das dimensões dos itens solicitados, podemos combiná-los de maneiras diferentes dentro de objetos (padrões de corte) a fim de atender à demanda, respeitando as restrições do processo de corte. Um conjunto de padrões de corte que atenda a uma demanda específica é denominado plano de corte, e é considerado ótimo se otimiza um certo critério, como, por exemplo, minimizar a perda de material (PILEGGI et al., 2005; SICUP, 2007).

Ao combinar itens dentro de objetos, dificilmente obtêm-se padrões de corte que utilizem todo o espaço disponível dos objetos. Isso resulta em sobras no plano de corte. Essas sobras podem ser consideradas perdas (sucatas) ou retalhos, dependendo de seus tamanhos. Define-se retalho como uma sobra de um objeto cortado que possua comprimento suficientemente grande para ser reaproveitada posteriormente para formar itens. Caso seja um retalho, a sobra pode ir para um estoque de objetos disponíveis, sendo reutilizada para atender a uma demanda futura como um objeto-retalho, e desta forma não é contabilizada como perda do padrão de corte. Convém ressaltar que a consideração de retalhos neste estudo configura um problema diferente do clássico problema de corte de estoque da literatura.

O planejamento dos padrões de corte em geral é uma das atividades importantes da programação de produção de uma empresa que utiliza parte de sua capacidade produtiva para cortar materiais para as demais etapas da produção. Essa atividade se relaciona com as demais atividades de planejamento e controle da produção (PCP). Assim, uma ferramenta eficaz que auxilie esta operação é de grande valia para melhorar os programas de produção e, conseqüentemente, a competitividade da empresa. O processo de corte para a produção de aeronaves leves, muitas vezes, resulta em perdas econômicas e desperdício de matériaprima. Há estimativas de que o metal estrutural da aeronave pode representar até $25 \%$ do seu custo final (NEIVA, 2006), o que torna importante um bom aproveitamento da matéria-prima necessária para produzir os pedidos.

Na empresa Indústria Aeronáutica Neiva Ltda. (Neiva), subsidiária da Empresa Brasileira de Aeronáutica S/A (Embraer), única fabricante de aviões agrícolas no País, onde estudamos esse problema, as perdas chegam 
à ordem de $52 \mathrm{~m} /$ dia (equivalente a aproximadamente $94 \mathrm{~kg}$ de liga metálica). Esses valores significam custos da ordem de US\$ $390 \mathrm{mil} / \mathrm{ano}$ em material específico fabricado com boa qualidade, vendido como sucata por não ter dimensões suficientes para uso prático na empresa. Uma parte dessas perdas é decorrente da ineficiência da programação da produção e pode ser evitada melhorando o planejamento dos padrões de corte, sem qualquer necessidade de investimento adicional nos equipamentos de corte. Parte da motivação para este trabalho é que essa aplicação foi pouco explorada na literatura, em particular nesse setor industrial.

Neste artigo, apresentamos dois modelos de programação linear inteira mista (modelos 1 e 2) com o objetivo de minimizar as perdas do material cortado neste processo de corte da empresa Neiva/Embraer, considerando a possibilidade de gerar retalhos. Os modelos são resolvidos por meio de uma linguagem de modelagem (GAMS) usando um software de otimização (CPLEX) (BROOKE et al., 1998). Para a validação dos modelos, experimentos computacionais foram realizados com dados reais do processo de corte da empresa, na qual não há utilização de nenhum programa "otimizador" para a formação dos planos de corte. Os resultados dos modelos 1 e 2 também foram comparados com as soluções obtidas por uma heurística residual de arredondamento guloso recentemente proposta na literatura, e com as soluções utilizadas pela empresa.

Na próxima seção, apresentamos uma breve descrição do processo produtivo e do problema de corte de estoque dentro deste processo. Na Seção 3, este problema de corte é classificado e a literatura relacionada é revisada. $\mathrm{Na}$ Seção 4, apresentamos os modelos 1 e 2 para apoiar as decisões do planejamento dos padrões de corte deste estudo de caso. Na Seção 5, são reportados os resultados dos experimentos computacionais realizados com os dois modelos, utilizando dados reais da empresa. Finalmente, na Seção 6, apresentamos as conclusões deste trabalho e discutimos perspectivas para pesquisa futura.

\section{A aviação agrícola, o processo produtivo e o problema de corte}

Registros do início de atividades agrícolas utilizando aeronaves leves datam do começo do século XX. Na Nova Zelândia, um agricultor semeou forrageiras (gramídeas) num terreno alagado com um balão de ar quente controlado por cordas (o dosador era um tubo com descarga controlada). Na Alemanha, um técnico florestal registrou, no Departamento de Patentes Imperial em Berlim, em 1911, um equipamento polvilhador para ser adaptado em aviões (Fokker bi-plano). Nos EUA, um avião aplicou inseticida num campo de algodão no estado da Louisiana, em 1917, utilizando uma polvilhadeira terrestre adap- tada em seu bojo. Há relato de que a aplicação foi 100 vezes mais rápida que a melhor máquina movida à força animal. Em 1958, começa a ser produzido, na empresa americana Piper Aircraft, o Pawnee, o primeiro avião especificamente agrícola fabricado em série e comercialmente (SILVEIRA, 2004).

No Brasil, a aviação agrícola teve sua origem em 1947 com um primeiro vôo agrícola acontecendo em Pelotas, RS. Este vôo sobrevoou uma área agrícola que sofria de ataques de gafanhotos, aplicando inseticida organoclorado (a aeronave era um biplano de madeira e tela, Muniz M-9, produzido pela Fábrica Brasileira de Aviões). A aviação agrícola contemporânea iniciou-se principalmente nos estudos realizados na fazenda Ipanema, do Ministério da Agricultura, localizada no município de Sorocaba, SP. Foi por essa razão que o Instituto Tecnológico da Aeronáutica (ITA) denominou Ipanema a aeronave nele projetada nos anos 60, que resultou na fabricação pela Neiva/Embraer do primeiro avião agrícola nacional, o EMB-200 Ipanema. Em 1974, um acordo de cooperação foi assinado com a Piper Aircraft, e a troca de tecnologias foi um impulso para a fabricação em larga escala do Ipanema. Nesse período, o Brasil já era o maior mercado de exportação de aviões leves dos EUA, superando o Canadá e a Alemanha.

A Neiva/Embraer ainda é a única empresa brasileira fabricante de uma aeronave exclusiva para o segmento agrícola e, atualmente, o Ipanema corresponde a aproximadamente $80 \%$ da frota nacional agrícola ativa. As demais aeronaves são importadas, especialmente dos EUA. Uma ilustração tradicional da aeronave Ipanema é apresentada na Figura 1. As principais utilizações de aeronaves leves voltadas para o segmento do mercado agrícola, referem-se a: a) pulverização eletrostática; b) difusor de sólidos; e c) demarcação via sistema de posicionamento global (differential global positioning service - DGPS). Pode-se também utilizar a aviação agrícola para: a) combate a vetores; b) combate a incêndios; c) povoamento de águas; d) semeadura; e e) aplicação de

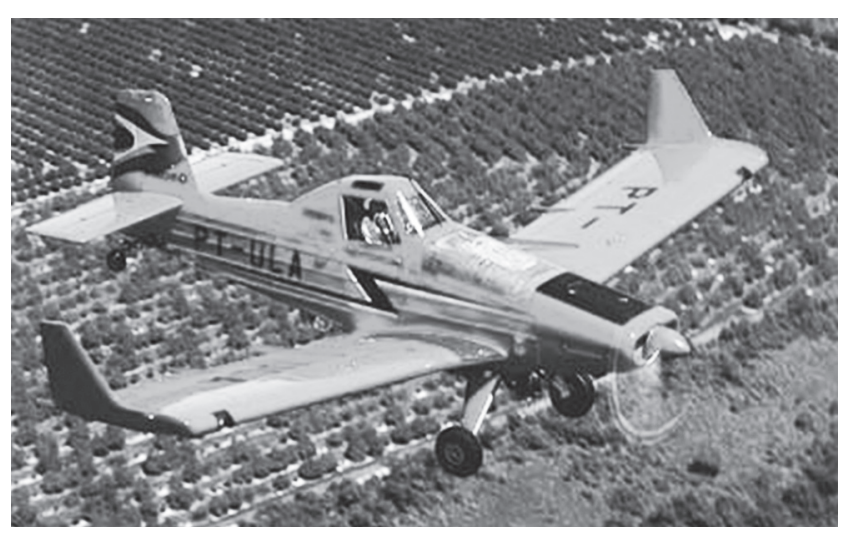

Figura 1. Imagem do Ipanema, 2007. (NEIVA, 2006). 
fertilizantes, defensivos agrícolas líquidos e sólidos, como herbicidas, fungicidas e inseticidas (NEIVA, 2006).

O planejamento da produção de aeronaves agrícolas baseia-se inicialmente na demanda das aeronaves. Forma-se, diária ou semanalmente, dentro do MRP (master resource planning; SLACK et al., 2002) da empresa, uma carteira de pedidos que deve ser cumprida por todos os processos de produção, começando pelo processo de corte de materiais. Nesse processo, uma abordagem de otimização poderia ser aplicada para definir os padrões de corte a serem utilizados para atender à demanda. A empresa Neiva/Embraer tem por política não considerar a formação de itens para estoque, sendo apenas cortado o requisitado pela etapa de montagem da aeronave, devido a requisitos de qualidade dos itens e rastreabilidade do material exigidos pela indústria aeronáutica. As etapas seguintes do sistema produtivo da aeronave, que não se aplicam neste estudo, são os processos de montagem das asas, da fuselagem e dos aviônicos, seguidos pela pintura, vôos de teste e entrega da aeronave.

A estrutura de uma aeronave leve é formada por pórticos treliçados - treliças que seguem os padrões estáticos de uma estrutura espacial, conforme é ilustrado na Figura 2. As treliças são compostas por peças - denominadas itens - oriundas do corte de objetos em estoque. Os itens são variados em comprimentos e especificações quanto à liga metálica, conforme o projeto. Estruturas espaciais são sistemas estaticamente indeterminados em alto grau, pois não são planas. Há resistências dos elementos quanto às solicitações dos esforços normais (tração e compressão), solicitações em flexão (flexão e força cortante) e solicitações combinadas. Essas estruturas na aeronave têm utilização interna e em parte externa, como é o caso do sistema de trem de pouso. A análise estrutural faz parte do projeto da aeronave, previamente dimensionado e aprovado, cabendo à produção apenas executá-lo conforme especificado em projeto. A principal justificativa da utilização dessa forma de construção é a leveza e a resistência, o que também a torna a opção mais viável economicamente.

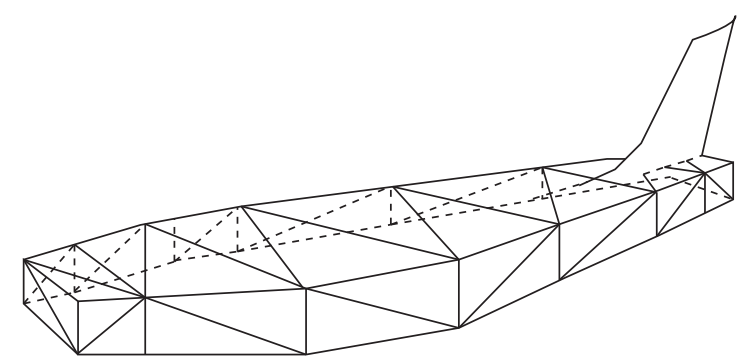

Figura 2. Idéia de uma estrutura tubular especial de uma aeronave.
A construção de aviões é realizada dessa maneira desde os princípios da aeronáutica. Antigamente, a estrutura estava à mostra e era em grande parte de madeira. Contudo, a utilização do aço na aviação se intensificou nos anos 40, quando as marinhas dos EUA e Grã-Bretanha começaram a exigir sua utilização em aeronaves de porta-aviões. Nos anos 60 , com o desenvolvimento de novos materiais, o aço obrigatório para partes estruturais de aeronaves passou a ser a liga cromo-molybdenum e o aço-carbono 4130 (THURSTON, 2000).

$\mathrm{O}$ presente problema de corte de estoque pode ser definido da seguinte maneira: encontrar um plano de corte que produza exatamente todos os itens demandados, gerando a menor perda possível e utilizando o menor comprimento (ou custo) de objetos. Em certos casos, as perdas dos padrões de corte escolhidos podem ser agrupadas em um único padrão, fato desejado pela empresa. Dessa forma, pode-se obter um comprimento suficientemente grande de tubo para posterior utilização (retalho). Esse problema pode ser caracterizado como um problema multi-objetivo: a) minimizar as perdas geradas pelos padrões de corte, que não têm tamanho suficiente para reaproveitamento; e b) minimizar o comprimento dos objetos em estoque utilizados, priorizando o corte dos objetos-retalhos em estoque.

A empresa Neiva/Embraer não possui um programa "otimizador" para determinar quais padrões de corte serão executados. A programação é realizada manualmente, com o auxílio de planilhas de cálculo, e baseada na experiência dos operadores que ali trabalham. O programador busca o aproveitamento máximo do comprimento de cada objeto cortado. Durante as visitas à empresa, notamos a estratégia de utilizar primeiro os objetos maiores, visto que há possibilidade de melhores combinações de itens dentro deles. Nesta estratégia, começa-se alocando primeiro os itens maiores aos objetos maiores, deixando os itens menores para o final, acreditando ser possível o melhor "encaixe" destes nas sobras dos cortes dos itens maiores. Notamos também uma tendência de tentar agregar itens de mesmo comprimento. Essa política da programação manual assemelha-se à heurística construtiva gulosa FFD (first fit decreasing; DYCKHOFF et al., 1985), que, grosso modo, consiste em ir alocando itens de maior comprimento primeiro, e em objetos de maior comprimento primeiro (no caso dos objetos terem tamanhos diferentes), e assim consecutivamente, respeitando a ordenação lexicográfica determinada, até que todos os itens estejam alocados ou que todos os objetos sejam utilizados, o que acontecer primeiro.

\section{Revisão da literatura}

Dyckhoff (1990) e Dyckhoff e Finke (1992) apresentaram uma tipologia fundamentada na estrutura lógica 
dos problemas de corte e empacotamento. Quatro características principais definem a tipologia: a) dimensões do problema; b) forma de alocação das unidades; c) objetivos; e d) restrições. A importância de definir estes tipos reside no fato de que estas características têm um impacto importante sobre a escolha e a complexidade dos métodos de solução. O problema de corte estudado neste trabalho, sem considerar a formação dos retalhos, pode ser classificado como 1/V/D/F: 1 indicando corte unidimensional; V (do alemão verladeproblem) indicando seleção de objetos e produção de todas os itens demandados; D (do inglês different) indicando que os objetos podem ter tamanhos diferentes; e $\mathrm{F}$ (do inglês few) indicando poucos itens, geralmente de tamanhos diferentes. Mais recentemente, Wäscher et al. (2007) estenderam a tipologia anterior com a introdução de novos critérios que definem outros problemas, além dos já definidos anteriormente. Segundo essa tipologia estendida, o presente problema de corte de estoque pode ser classificado como um problema de corte residual (residual cutting stock problem - RCSP) e, dentro dessa categoria, como um problema de corte unidimensional híbrido (hybrid one-dimensional cutting stock problem - HODCSP).

Não existem métodos de solução gerais e eficientes para os problemas de corte e empacotamento devido, principalmente, à complexidade computacional dos problemas e à diversidade de casos em que estes podem aparecer. Os modelos que descrevem problemas reais são, na maioria das vezes, complexos, com várias restrições e variáveis. Como os problemas são em geral NP-difíceis, resolvê-los, encontrando uma solução suficientemente boa e rápida, pode ser uma tarefa difícil (GAREY; JOHNSON, 1979; ARENALES et al., 1999). Três possíveis abordagens de solução para os problemas são: a) programação linear com um procedimento de geração de colunas (GILMORE; GOMORY, 1961, 1963) e com um procedimento de arredondamento da solução (POLDI, 2003); b) programação inteira mista (PIM) (LASDON, 1970; WILLIAMS, 1978; DYCKHOFF, 1981); e, ainda, c) heurísticas e metaheurísticas (STADTLER, 1990, ARMBRUSTER, 2002; ALVIM et al., 2004).

Uma razão que não encorajou a utilização da abordagem a) no presente problema de corte de estoque é o fato da demanda de alguns itens ser muito pequena (por exemplo, a demanda de vários itens é unitária), o que dificulta a aplicação da relaxação linear do problema de corte de estoque, mesmo considerando o emprego de técnicas de arredondamento da solução. Outra dificuldade é o fato da oferta de alguns objetos também ser muito pequena (por exemplo, apenas uma unidade disponível de cada objeto-retalho), implicando que o número de vezes que um possível padrão de corte pode ser executado nesses objetos não pode ser maior que 1 , o que também dificulta $\mathrm{o}$ arredondamento da solução da relaxação linear.
Um exemplo da abordagem b) é o modelo de PIM de Dyckhoff (1981) para problemas de corte de estoque unidimensional. Usando uma estrutura denominada one-cuts para representar um corte, o modelo considera cortar cada objeto em dois pedaços, sendo que pelo menos um deles tem comprimento igual ao de um item demandado. $\mathrm{O}$ outro pedaço, caso também não corresponda a um item demandado, mas possua comprimento suficiente para produzir itens, retorna para o estoque como objeto-retalho. Note que, estes pedaços residuais (retalhos) podem ser utilizados para produzir itens, ou seja, o modelo considera o reaproveitamento do material.

Uma dificuldade para utilizar o modelo de Dyckhoff (1981) no presente trabalho é que as restrições do modelo não limitam o número de retalhos gerados, o que é desejável na presente aplicação. Um modelo matemático mais diretamente relacionado com o problema de corte de estoque do presente trabalho foi proposto em Gradisar et al. (1997). O modelo foi apresentado para representar um problema de corte de estoque de rolos de tecido, mas não foi utilizado para resolvê-lo. Ao contrário, naquele estudo e em estudos posteriores (GRADISAR et al., 1999a, 1999b, 2002), heurísticas construtivas foram propostas para resolver o problema, devido às dificuldades associadas à modelagem e ao grande número de variáveis e restrições envolvidos. Embora não tenha sido explicitamente formulado como um PIM, o modelo pode ser reescrito como um PIM (conforme mostrado na Seção 4) e, portanto, pode ser visto como um exemplo da abordagem b) acima.

No problema estudado por Gradisar et al. (1997), moldes de peças de roupas são combinadas dentro de retângulos e formam carteiras de pedidos de itens que se diferenciam uns dos outros em apenas uma dimensão, a longitudinal. Estes itens devem ser cortados unidimensionalmente a partir dos rolos de tecido para, então, em pedaços menores, serem repassados para costureiras, que fazem o corte bidimensional do tecido e montam as peças de roupas. O objetivo é minimizar as perdas resultantes do corte dos rolos de tecido. A peculiaridade desse problema é que todos os rolos de tecido, no caso os objetos, têm tamanhos diferentes em decorrência do processo de fabricação desse material. Assim como o presente problema de corte de tubos, no problema de corte de rolos de tecidos também é possível armazenar as sobras com comprimentos suficientemente grandes para posterior utilização (retalhos). Além disso, a demanda deve ser cumprida caso haja recursos suficientes; caso contrário, o modelo deve minimizar os itens não produzidos.

No problema estudado neste trabalho, admite-se que sempre haverá objetos suficientes para a produção de todos os itens demandados, uma vez que a Neiva/ Embraer mantém estoques suficientemente grandes desses objetos para evitar a falta de material no processo 
de corte. Também, admite-se que os tempos de preparação dos padrões de corte no equipamento de corte não são relevantes, quando comparados com os tempos totais de produção dos padrões.

\subsection{Modelo de Gradisar et al. (1997)}

O modelo a seguir é uma simples adaptação do modelo de Gradisar et al. (1997) sob a condição de não haver limites de alocação de diferentes itens por objeto. Considere que os itens do tipo $\mathrm{i}, \mathrm{i}=1,2, \ldots, \mathrm{m}$, com comprimento $1_{i}$ e demanda $d_{i}$, devem ter suas demandas exatamente satisfeitas a partir dos objetos $\mathrm{j}, \mathrm{j}=1,2, \ldots, \mathrm{n}$, com comprimento $b_{j}$. Admite-se que $l_{i}, d_{i}$ e $b_{j}$ sejam números inteiros. A variável de decisão $\mathrm{x}_{\mathrm{ij}}$ indica se um item do tipo i é alocado $\left(\mathrm{x}_{\mathrm{ij}}=1\right)$ ou não $\left(\mathrm{x}_{\mathrm{ij}}=0\right)$ no objeto $\mathrm{j}$. Seja $\mathrm{N} \mathrm{o}$ comprimento mínimo aceito pela empresa como retalho. A Equação 1 garante que, para todo objeto j, o comprimento total de itens $\mathrm{i}$ alocados no objeto $\mathrm{j}$ não exceda o comprimento do objeto. Caso o comprimento dos itens não seja exatamente igual ao comprimento do objeto, então a variável de folga não-negativa $\delta_{\mathrm{j}}$ é igual a essa diferença, para todo $\mathrm{j}$.

$$
\sum_{i=1}^{m} l_{i} \cdot x_{i j}+\delta_{j}=b_{j} \quad \forall j
$$

A Equação 2 garante que, para todo tipo de item i, o total de itens cortados dos objetos seja igual à demanda. Caso contrário, essa diferença é igual à variável de folga não-negativa $\Delta \mathrm{i}$, para todo $\mathrm{i}$.

$$
\sum_{j=1}^{n} x_{i j}+\Delta_{\mathrm{i}}=d_{i} \quad \forall i
$$

A variável $z_{j}$ indica se o objeto j é utilizado $\left(z_{j}=1\right)$ ou não $\left(\mathrm{z}_{\mathrm{j}}=0\right)$ no plano de corte, conforme definido na Equação 3:

$$
z_{j}=\left\{\begin{array}{l}
1 \text { se } \sum_{i=1}^{m} x_{i j}>0, \quad \forall j \\
0 \text { se } \sum_{i=1}^{m} x_{i j}=0, \quad \forall j
\end{array}\right.
$$

Caso o objeto $\mathrm{j}$ esteja no plano de corte, a variável $\mathrm{t}_{\mathrm{j}}$ corresponde a sobra $\delta_{\mathrm{j}}$ do padrão de corte do objeto $\mathrm{j}$, se essa sobra for uma perda (isto é, menor do que N). A Equação 4 garante isso para todo $\mathrm{j}$ :

$$
t_{j}=\left\{\begin{array}{lll}
\delta_{j} & \text { se } z_{j}=1 & \text { e } \delta_{j}<N \quad \forall j \\
0 & \text { se } z_{j}=0 & \text { ou } \delta_{j} \geq N \quad \forall j
\end{array}\right.
$$

Se um objeto $\mathrm{j}$ está no plano de corte, a variável $\mathrm{u}_{\mathrm{j}}$ indica se a sobra do objeto $\mathrm{j}$ é um retalho $\left(\mathrm{u}_{\mathrm{j}}=1\right)$ ou não $\left(\mathrm{u}_{\mathrm{j}}=0\right)$, isto é, se a sobra é maior ou igual a $\mathrm{N}$ ou não. A Equação 5 garante isso para todo $\mathrm{j}$ :

$$
u_{j}=\left\{\begin{array}{l}
1 \text { se } z_{j}=1 \text { e } \delta_{j} \geq N \quad \forall j \\
0 \text { se } z_{j}=0 \text { ou } \delta_{j}<N \quad \forall j
\end{array}\right.
$$

Note que é preciso limitar no modelo o número máximo de retalhos produzidos, caso contrário, retalhos podem ser superproduzidos apenas para manter padrões de corte sem perda. A Equação 6 limita este número a 1 (outros valores poderiam ter sido usados ao invés de 1):

$$
\sum_{j=1}^{n} u_{j} \leq 1
$$

O modelo tem dois objetivos. A Equação 7 define o objetivo de minimizar os itens não fabricados:

$$
F O_{1}=\min \sum_{i=1}^{m} \Delta_{\mathrm{i}}
$$

E a Equação 8 define o objetivo de minimizar a perda do material. Essa perda se refere apenas às sobras menores do que o comprimento $\mathrm{N}$, já que as sobras maiores ou iguais a este valor são consideradas retalhos.

$$
F O_{2}=\min \sum_{j=1}^{n} t_{j}
$$

Como estamos admitindo que $1_{\mathrm{i}}, \mathrm{d}_{\mathrm{i}}$ e $\mathrm{b}_{\mathrm{j}}$ são números inteiros, segue que $\Delta_{\mathrm{i}}$ e $\mathrm{t}_{\mathrm{j}}$ também são números inteiros, e, portanto, $\mathrm{FO}_{1}$ e $\mathrm{FO}_{2}$ nas Equações 7 e 8 sempre resultam em valores inteiros. Os domínios das variáveis do modelo são:

$$
\begin{aligned}
& \mathrm{x}_{\mathrm{ij}} \geq 0 \text { e inteiro } \forall_{\mathrm{ij}}, \delta_{\mathrm{j}} \geq 0 \forall \mathrm{j}, \mathrm{t}_{\mathrm{j}} \geq 0 \forall \mathrm{j}, \\
& \mathrm{z}_{\mathrm{j}} \in\{0,1\} \forall \mathrm{j}, \mathrm{u}_{\mathrm{j}} \in\{0,1\} \forall \mathrm{j}, \Delta_{\mathrm{i}} \geq 0 \forall \mathrm{i}
\end{aligned}
$$

Note que o modelo matemático acima não pode ser diretamente implementado em uma linguagem de modelagem e resolvido por um software de otimização, uma vez que não está escrito como um modelo de PIM. Na Seção 4 a seguir, isso é realizado (modelo 1) para verificar se esta abordagem resolve otimamente o modelo em um tempo computacional aceitável para o problema de corte de estoque da empresa Neiva/Embraer. Também é proposto um segundo modelo (modelo 2), alternativo ao modelo 1.

\section{Modelagem matemática}

A modelagem matemática considera que o planejamento do corte dos objetos deve ser realizado em um único período de planejamento, em uma única máquina de corte, e admite que a quantidade de objetos disponíveis em estoque é suficientemente grande e os tempos de preparação dos padrões de corte na máquina não são relevantes.

\section{Modelo 1}

Conforme mencionado na Seção 3, o modelo 1 corresponde ao modelo de Gradisar et al. (1997) descrito como um modelo de PIM. A equação (1) é considerada da mesma forma. A Equação 2 é reescrita conforme na Equação 9, de 
forma que a demanda de todos os itens seja exatamente satisfeita, pois se admite que exista um número $\mathrm{n}$ suficientemente grande de objetos disponíveis em estoque.

$$
\sum_{j=1}^{n} x_{i j}=d_{i} \quad \forall i
$$

A Equação 3 é descrita pelas Equações 10 e 11, em que $M$ é um número suficientemente grande (por exemplo, $\left.\mathrm{M}=\max _{\mathrm{j}}\left\{\mathrm{b}_{\mathrm{j}}\right\}-\min _{\mathrm{i}}\left\{\mathrm{l}_{\mathrm{i}}\right\}\right)$.

$$
\begin{gathered}
z_{j} \leq \sum_{i} x_{i j} \quad \forall j \\
\sum_{i} x_{i j} \leq M . z_{j} \quad \forall j
\end{gathered}
$$

Para definir a variável $t_{j}$ na Equação 4, define-se uma variável binária auxiliar $\mathrm{w}_{\mathrm{j}}$ que indica quando $\delta_{\mathrm{j}}<\mathrm{N}$ $\left(\mathrm{w}_{\mathrm{j}}=1\right)$ e quando $\delta_{\mathrm{j}} \geq \mathrm{N} \quad\left(\mathrm{w}_{\mathrm{j}}=0\right)$. As Equações 12 e 13 relacionam isso:

$$
\begin{aligned}
& \left(\delta_{j}-N\right) \geq-M . w_{j}+\varepsilon \forall j \\
& \left(\delta_{j}-N\right) \leq M .\left(1-w_{\mathrm{j}}\right) \forall j
\end{aligned}
$$

Em que $\varepsilon$ é um número positivo pequeno para mudar o sinal das desigualdades de " $\geq$ " para " $>$ ". Isso é um simples artifício para codificar estas desigualdades em linguagens de modelagem. No caso de todos os parâmetros de entrada serem números inteiros, então $\varepsilon=1$.

Definido nas Equações 12 e 13 o valor da variável binária auxiliar $\mathrm{w}_{\mathrm{j}}$, caso a sobra $\left(\delta_{\mathrm{j}}\right)$ do corte dos itens nesse objeto seja menor que o valor de $\mathrm{N}\left(\mathrm{w}_{\mathrm{j}}=1\right)$, e também esse objeto $\mathrm{j}$ esteja presente no plano de corte escolhido $\left(z_{j}=1\right)$, a variável $t_{j}$, pela Equação 4, assume o valor da sobra $\delta_{j}$ do padrão de corte do objeto $\mathrm{j}$ (i.e., $\mathrm{t}_{\mathrm{j}}=\delta_{\mathrm{j}}$ ). Caso contrário, para determinado $\mathrm{j}$ em que $\mathrm{z}_{\mathrm{j}}=0$ ou $\mathrm{w}_{\mathrm{j}}=0$, tem-se que $\mathrm{t}_{\mathrm{j}}=0$. Lembre-se que a soma de $t_{j}$ (para todos os objetos, $\mathrm{j}=1$ até $n$ ) é minimizado na função objetivo do modelo. Reescrevendo em termos de funções lineares a Equação 4, além de (12) e (13), temos as Equações 14, 15, 16 e 17 (para mais detalhes destas e de outras descrições a seguir, veja, por exemplo, Williams, 1978).

$$
\begin{gathered}
t_{j}-M \cdot w_{j} \leq 0 \forall j \\
t_{j}-M \cdot z_{j} \leq 0 \forall j \\
-\delta_{j}+t_{j} \leq 0 \forall j \\
\delta_{j}-t_{j} \leq 0 \forall j
\end{gathered}
$$

Para utilizar a variável $u_{\mathrm{j}}$ na Equação 5 no modelo 1, é necessário também definir uma outra variável binária auxiliar yj para indicar quando $\delta_{j} \geq \mathrm{N}\left(\mathrm{y}_{\mathrm{j}}=1\right)$ e quando
$\delta_{\mathrm{j}}<\mathrm{N}\left(\mathrm{y}_{\mathrm{j}}=0\right)$. Similarmente às restrições (12) e (13) para $\mathrm{w}_{\mathrm{j}}$, as Equações 18 e 19 relacionam isso:

$$
\begin{gathered}
\left(\delta_{j}-N\right) \leq M \cdot y_{j} \forall j \\
\left(\delta_{j}-N\right) \geq M \cdot\left(y_{j}-1\right)+\varepsilon \forall j
\end{gathered}
$$

Definido nas Equações 18 e 19 o valor da variável binária auxiliar yj, caso a sobra $\left(\delta_{j}\right)$ do corte dos itens nesse objeto seja maior ou igual ao valor de $\mathrm{N}\left(\mathrm{y}_{j}=1\right)$, e também esse objeto $\mathrm{j}$ esteja presente no plano de corte escolhido $\left(\mathrm{z}_{j}=1\right)$, a variável $\mathrm{u}_{\mathrm{j}}$, pela Equação 5 , assume o valor $1\left(u_{j}=1\right)$. Caso contrário, para determinado $\mathrm{j}$ em que $\mathrm{z}_{j}=0$ ou $\mathrm{y}_{j}=0$, tem-se pela Equação 5 que $\mathrm{u}_{j}=0$. Reescrevendo em termos de funções lineares a Equação 5, temos, além das Equações 18 e 19, também as Equações 20, 21 e 22:

$$
\begin{gathered}
-z_{j}+u_{j} \leq 0 \forall j \\
-y_{j}+u_{j} \leq 0 \forall j \\
z_{j}+y_{j}-u_{j} \leq 1 \forall j
\end{gathered}
$$

Note que as equações de 12 a 22 juntas descrevem as Equações 4 e 5 do modelo anterior. A Equação 6, que limita o número de retalhos possíveis de serem gerados, é considerada da mesma forma.

Somente a função objetivo $\mathrm{FO}_{2}$ (Equação 8) é considerada no modelo 1 , dado que admite-se que $\mathrm{n}$ seja um número suficientemente grande para produzir todos os itens demandados. Lembre-se que essa função objetivo minimiza a perda dos padrões de corte, isto é, as sobras com comprimentos menores do que $\mathrm{N}$, já que as sobras maiores não são consideradas perdas, mas sim retalhos. Para o presente trabalho, $\mathrm{N}$ foi considerado igual ao comprimento do menor item da carteira de pedidos de cada exemplo executado, isto é, $M=\max _{j}\left\{b_{j}\right\}-\min _{i}\left\{l_{i}\right\}$ e $N=\min _{i}\left\{l_{i}\right\}$.

Assim, o modelo 1 é definido pelas Equações 1, 6 e 8-22. Os domínios das variáveis do modelo 1 são:

$$
\begin{gathered}
\mathrm{x}_{\mathrm{ij}} \geq 0 \text { e inteiro } \forall \mathrm{ij}, \delta_{\mathrm{j}} \geq 0 \forall \mathrm{j}, \mathrm{t}_{\mathrm{j}} \geq 0 \forall \mathrm{j}, \\
\mathrm{z}_{\mathrm{j}} \in\{0,1\} \forall \mathrm{j}, \mathrm{y}_{\mathrm{j}} \in\{0,1\} \forall \mathrm{j}, \mathrm{u}_{\mathrm{j}} \in\{0,1\} \forall \mathrm{j}
\end{gathered}
$$

\section{Modelo 2}

O modelo 2 pode ser visto como uma simplificação do modelo 1. As Equações 1, 6 e 9 e a função objetivo 8 são consideradas da mesma forma que anteriormente.

Lembre-se que a variável $z_{\mathrm{j}}$ indica se o objeto $\mathrm{j}$ é utilizado $\left(\mathrm{z}_{\mathrm{j}}=1\right)$ ou não $\left(\mathrm{z}_{\mathrm{j}}=0\right)$ no plano de corte. Caso o valor da diferença entre o comprimento do objeto $\mathrm{j}$ e a soma dos comprimentos dos itens alocados ao objeto seja maior do que o valor $\mathrm{N}$, então a variável $\mathrm{u}_{\mathrm{j}}$ pode ser igual a 1 , caso contrário, ela é necessariamente igual a 0 . Essas condições estão descritas na Equação 23: 


$$
N . u_{j} \leq b_{j} \cdot z_{j}-\sum_{i=1}^{m} l_{i} \cdot x_{i j} \quad \forall j
$$

Caso um objeto j esteja no plano de corte escolhido $\left(\mathrm{z}_{\mathrm{j}}=1\right) \mathrm{e}$, necessariamente, se tenha $\mathrm{u}_{\mathrm{j}}$ igual a 0 (Equação 23), pela Equação 24, determina-se a diferença entre o comprimento do objeto $\mathrm{j}$ e a soma de todos os comprimentos dos itens alocados nesse objeto como sendo igual a $\mathrm{t}_{\mathrm{j}}$. Caso contrário, $\mathrm{t}_{\mathrm{j}}=0$.

$$
b_{j} \cdot z_{j}-\sum_{i=1}^{m} l_{i} \cdot x_{i j} \leq t_{j}+u_{j} \cdot M \quad \forall j
$$

Assim, o modelo 2 é definido pelas Equações 1, 6, 8, 9, 23 e 24 . As definições de $M$ e $N$ são iguais às do modelo 1. Os domínios das variáveis do modelo 2 são:

$$
\mathrm{x}_{\mathrm{ij}} \geq 0 \text { e inteiro } \forall \mathrm{ij}, \mathrm{t}_{\mathrm{j}} \geq 0 \forall \mathrm{j}, \mathrm{z}_{\mathrm{j}} \in\{0,1\} \forall \mathrm{j}, \mathrm{u}_{\mathrm{j}} \in\{0,1\}
$$
$\forall \mathrm{j}$.

Convém observar que a função objetivo (Equação 8) dos modelos 1 e 2 pode ser ligeiramente modificada para também considerar o critério de minimizar o comprimento total cortado de objetos (veja Seção 2). Para soluções alternativas de perda mínima, a idéia é favorecer aquelas que evitam a geração de novos retalhos. Neste caso, a Equação 8 deve ser substituída pela Equação 25:

$$
F O_{3}=\min \sum_{j=1}^{n} t_{j}+\frac{\sum_{j=1}^{n} b_{j} z_{j}}{\sum_{j=1}^{n} b_{j}}
$$

Dado que os parâmetros de entrada dos modelos são supostos números inteiros, o primeiro termo de $\mathrm{FO}_{3}$ (i.e., a perda total) é um número inteiro. Portanto, como o segundo termo sempre resulta em um valor maior que 0 e menor que 1, este termo serve para desempatar soluções alternativas de perda mínima em favor das soluções que minimizem o comprimento total cortado de objetos.

Outra possível modificação nos modelos 1 e 2 é relaxar a Equação 6, que limita o número máximo de retalhos, e incluí-la na função objetivo Equação 8 com um multiplicador (penalização), para também considerar o critério de minimizar o número total de retalhos gerados. Desta maneira, os modelos estariam considerando o trade-off entre os objetivos de minimizar a perda de material e o número de retalhos gerados. Neste caso, a Equação 8 deve ser substituída pela Equação 26:

$$
F O_{4}=\min \sum_{j=1}^{n} t_{j}+\alpha \sum_{j=1}^{n} u_{j}
$$

Sendo $\alpha$ o peso deste critério. Por exemplo, definindo-se $\alpha=1 / \mathrm{n}$, o segundo termo de $\mathrm{FO}_{4}$ sempre resultaria entre 0 e 1 , e a idéia seria favorecer soluções que gerassem o mínimo de retalhos possível, dentre as soluções alternativas de perda mínima.
Embora interessantes, nenhuma destas duas possíveis modificações em $\mathrm{FO}_{2}$ foram consideradas nos experimentos da seção a seguir.

\section{Resultados computacionais}

Para resolver os modelos 1 e 2 apresentados na Seção 4, foram utilizados a linguagem de modelagem GAMS 19.6 e o solver comercial CPLEX 7.0, em um microcomputador com processador Intel Pentium III com $550 \mathrm{Mhz}$ e $512 \mathrm{Mb}$ de memória RAM. Nos experimentos, foram utilizados os parâmetros default do GAMS e do CPLEX, exceto o gap de otimalidade que foi fixado em zero e o limite do número de iterações do solver, que foi relaxado. O tempo de execução para cada exemplo foi limitado em uma hora, valor escolhido arbitrariamente e considerado razoável para apoiar as decisões envolvidas na prática da empresa. Quando não foi possível encontrar uma solução ótima pelos modelos dentro desse tempo estipulado, a melhor solução inteira obtida foi reportada e assinalada com asterisco.

\section{Experimento 1}

Este experimento comparou as soluções obtidas pelo GAMS/CPLEX com os modelos 1 e 2 e as soluções conseguidas pela programação manual da empresa, realizadas com o auxílio de uma planilha de cálculo. Com os dois modelos, resolveram-se 50 exemplos que formam parte da demanda de fabricação da aeronave Ipanema. Nesses exemplos, a quantidade de itens varia entre 7 e 120 itens e a quantidade de tipos de itens (m) varia entre 2 e 34 tipos de itens, com comprimentos $1_{i}$ variando de 30 até $2320 \mathrm{~mm}$. Os comprimentos padrões $b_{j}$ dos objetos em estoque podem ser de três tamanhos diferentes: $6.000,3.500$ ou $3.000 \mathrm{~mm}$. Cada exemplo utiliza apenas um destes tamanhos, definido a priori em função das diferentes especificações de material (por exemplo, a espessura da parede dos tubos) e também em função do fornecedor da matéria-prima e do lote de fabricação (por questões de rastreabilidade de material). Ou seja, todos os $\mathrm{n}$ objetos disponíveis de cada exemplo têm o mesmo comprimento (assumimos que as quantidades em estoque deste comprimento são suficientemente grandes para produzir toda demanda, o que é razoável na prática). A descrição completa destes exemplos encontra-se em Abuabara (2006).

Na Tabela 1, são apresentados os resultados dos 50 exemplos obtidos pela programação manual e para os modelos 1 e 2 . A tabela está dividida em três colunas principais de acordo com a abordagem de solução (Programação Manual, Modelo 1 e Modelo 2). Para cada abordagem, a tabela apresenta três subcolunas que representam na solução: o número de objetos de comprimento padrão utilizados (Objetos); o número de retalhos gerados 
Tabela 1. Resultados do Experimento 1, solução de 50 exemplos da carteira de pedidos da aeronave Ipanema.

\begin{tabular}{|c|c|c|c|c|c|c|c|c|c|}
\hline \multirow[b]{2}{*}{ Ex. } & \multicolumn{3}{|c|}{ Programação Manual } & \multicolumn{3}{|c|}{ Modelo 1} & \multicolumn{3}{|c|}{ Modelo 2} \\
\hline & Objetos & Retalhos & Perda $(\%)$ & Objetos & Retalhos & Perda $(\%)$ & Objetos & Retalhos & Perda $(\%)$ \\
\hline 1 & 2 & 1 & 1,57 & 2 & 1 & 1,57 & 2 & 1 & 1,57 \\
\hline 2 & 3 & 1 & 0,69 & 3 & 1 & 0,69 & 3 & 1 & 0,69 \\
\hline 3 & 4 & 1 & 1,30 & 4 & 1 & 0,24 & 4 & 1 & 0,24 \\
\hline 4 & 2 & 1 & 1,83 & 2 & 1 & 0,83 & 2 & 1 & 0,83 \\
\hline 5 & 3 & 1 & 3,67 & 3 & 1 & 0,67 & 3 & 1 & 0,67 \\
\hline 6 & 4 & 1 & 6,04 & 4 & 1 & 3,06 & 4 & 1 & 3,06 \\
\hline 7 & 4 & 1 & 0,72 & 4 & 1 & $* 0,01$ & 4 & 1 & 0,00 \\
\hline 8 & 2 & 1 & 2,75 & 2 & 1 & 0,04 & 2 & 1 & 0,04 \\
\hline 9 & 3 & 1 & 0,67 & 3 & 1 & 0,00 & 3 & 1 & 0,00 \\
\hline 10 & 2 & 1 & 0,21 & 2 & 1 & 0,00 & 2 & 1 & 0,00 \\
\hline 11 & 2 & 1 & 0,25 & 2 & 1 & 0,00 & 2 & 1 & 0,00 \\
\hline 12 & 3 & 1 & 0,36 & 3 & 1 & 0,00 & 3 & 1 & 0,00 \\
\hline 13 & 4 & 2 & 0,31 & 4 & 1 & 0,00 & 4 & 1 & 0,00 \\
\hline 14 & 6 & 1 & 0,37 & 6 & 1 & $* 0,05$ & 6 & 1 & 0,00 \\
\hline 15 & 2 & 1 & 6,57 & 2 & 1 & 3,14 & 2 & 1 & 3,14 \\
\hline 16 & 3 & 1 & 8,95 & 3 & 1 & 4,19 & 3 & 1 & 4,19 \\
\hline 17 & 4 & 1 & 6,57 & 4 & 1 & 6,57 & 4 & 1 & 6,57 \\
\hline 18 & 4 & 0 & 10,00 & 4 & 1 & 4,71 & 4 & 1 & 4,71 \\
\hline 19 & 7 & 5 & 0,08 & 6 & 1 & $* 0,07$ & 6 & 1 & 0,00 \\
\hline 20 & 3 & 3 & 0,00 & 3 & 1 & 0,05 & 3 & 1 & 0,05 \\
\hline 21 & 5 & 4 & 0,00 & 5 & 1 & $* 0,06$ & 5 & 1 & 0,00 \\
\hline 22 & 3 & 2 & 0,10 & 3 & 2 & 0,00 & 3 & 2 & 0,00 \\
\hline 23 & 6 & 4 & 0,10 & 6 & 3 & 0,19 & 6 & 3 & 0,19 \\
\hline 24 & 3 & 1 & 1,39 & 3 & 1 & 1,39 & 3 & 1 & 1,39 \\
\hline 25 & 4 & 3 & 1,75 & 4 & 1 & 1,75 & 4 & 1 & 1,75 \\
\hline 26 & 4 & 3 & 1,63 & 4 & 1 & 1,63 & 4 & 1 & 1,63 \\
\hline 27 & 2 & 1 & 1,00 & 2 & 1 & 0,13 & 2 & 1 & 0,13 \\
\hline 28 & 4 & 1 & 2,14 & 3 & 0 & 0,62 & 3 & 0 & 0,62 \\
\hline 29 & 4 & 1 & 0,25 & 4 & 1 & 0,10 & 4 & 1 & 0,10 \\
\hline 30 & 3 & 1 & 0,47 & 3 & 1 & 0,00 & 3 & 1 & 0,00 \\
\hline 31 & 6 & 1 & 0,39 & 6 & 2 & 0,00 & 6 & 2 & 0,00 \\
\hline 32 & 2 & 1 & 2,17 & 2 & 1 & 0,00 & 2 & 1 & 0,00 \\
\hline 33 & 4 & 1 & 2,92 & 4 & 2 & 0,00 & 4 & 2 & 0,00 \\
\hline 34 & 2 & 1 & 0,07 & 2 & 1 & 0,07 & 2 & 1 & 0,07 \\
\hline 35 & 3 & 3 & 0,00 & 3 & 1 & 0,10 & 3 & 1 & 0,10 \\
\hline 36 & 4 & 4 & 0,00 & 4 & 1 & 0,11 & 4 & 1 & 0,11 \\
\hline 37 & 2 & 1 & 4,00 & 2 & 1 & 0,00 & 2 & 1 & 0,00 \\
\hline 38 & 4 & 1 & 4,48 & 4 & 1 & 2,00 & 4 & 1 & 2,00 \\
\hline 39 & 6 & 1 & 4,52 & 5 & 0 & $* 1,94$ & 5 & 0 & 1,94 \\
\hline 40 & 2 & 1 & 3,08 & 2 & 1 & 3,08 & 2 & 1 & 3,08 \\
\hline 41 & 2 & 1 & 3,33 & 2 & 1 & 0,08 & 2 & 1 & 0,08 \\
\hline 42 & 2 & 1 & 1,92 & 3 & 1 & 1,28 & 3 & 1 & 1,28 \\
\hline 43 & 3 & 1 & 1,22 & 3 & 1 & 0,28 & 3 & 1 & 0,28 \\
\hline 44 & 4 & 2 & 2,50 & 4 & 1 & 2,00 & 4 & 1 & 2,00 \\
\hline 45 & 3 & 1 & 0,69 & 3 & 1 & 0,69 & 3 & 1 & 0,69 \\
\hline 46 & 6 & 3 & 5,00 & 5 & 1 & 1,39 & 5 & 1 & 1,39 \\
\hline
\end{tabular}

** Considerando apenas os exemplos com soluções com certificado de otimalidade dentro do tempo estipulado. 
Tabela1. Continuação...

\begin{tabular}{lccccccccc}
\hline \multicolumn{4}{c}{ Programação Manual } & \multicolumn{3}{c}{ Modelo 1 } & \multicolumn{2}{c}{ Modelo 2 } \\
\hline Ex. & Objetos & Retalhos & Perda & Objetos & Retalhos & Perda & Objetos & Retalhos & Perda \\
\hline 47 & 4 & 1 & 1,25 & 4 & 1 & 0,00 & 4 & 1 & 0,00 \\
48 & 5 & 2 & 3,63 & 4 & 1 & 0,37 & 4 & 1 & 0,37 \\
49 & 7 & 2 & 4,00 & 6 & 1 & $* 0,16$ & 6 & 1 & $* 0,16$ \\
50 & 6 & 1 & 3,18 & 6 & 1 & 2,87 & 6 & 1 & 2,87 \\
Total & 182 & 77 & - & 177 & 53 & - & 177 & 53 & - \\
Média & 3,64 & 1,54 & 2,20 & 3,54 & 1,06 & $0,96(* * 1,04)$ & 3,54 & 1,06 & $0,96(* * 0,98)$ \\
Desvio & 1,44 & 1,05 & 2,36 & 1,27 & 0,42 & $1,44(* * 1,50)$ & 1,27 & 0,42 & $1,44(* * 1,45)$ \\
\hline
\end{tabular}

** Considerando apenas os exemplos com soluções com certificado de otimalidade dentro do tempo estipulado.

(Retalhos); e a porcentagem da perda (Perda). A perda foi calculada dividindo-se o comprimento total descartado pelo comprimento total cortado em cada exemplo. Nas duas últimas linhas da tabela, observam-se as médias dos valores das colunas e os respectivos desvios padrões.

Todas as soluções encontradas com o modelo 2 utilizando o GAMS/CPLEX obtiveram certificado de otimalidade dentro do tempo limite estipulado, com exceção do exemplo 49. No caso do modelo 1 , as soluções encontradas para os exemplos 7, 14, 19 e 21 não são ótimas (embora sejam próximas da ótima), e as soluções encontradas para os exemplos 39 e 49 não obtiveram certificado de otimalidade, dentro do tempo limite estipulado utilizando o GAMS/CPLEX. Estes exemplos estão indicados na Tabela $1 \mathrm{com}$ um asterisco. Estes resultados mostram que o modelo 2 tem um desempenho melhor do que o modelo 1 neste experimento. Nos demais exemplos, as soluções dos dois modelos têm o mesmo número de retalhos gerados, o mesmo número de objetos utilizados e as mesmas perdas calculadas. Em média, os modelos obtiveram soluções bem próximas, e cerca de $65 \%$ melhores que as soluções da programação manual $(2,89 \%$ vs. aproximadamente $1 \%$ ). Os modelos também geraram aproximadamente $31 \%$ menos retalhos do que a programação manual (77 vs. 53) e utilizaram 3\% menos matéria-prima (182 vs. 177), o que é significativo na prática.

Convém observar em ambos os modelos que, em função da Equação 6, esses consideram como padrão no máximo a geração de uma sobra como retalho. Observando a Tabela 1, no caso da solução obtida envolver mais de um comprimento como retalho, temos: a) para o modelo 1, esses exemplos não teriam uma solução factível encontrada pelo solver; então, relaxou-se a restrição, permitindo-se a geração de um retalho a mais e, assim consecutivamente, até que tais exemplos fossem resolvidos pelo solver, obtendo-se soluções factíveis; e b) para o modelo 2, não se alterou a restrição, pois todas as sobras (além de uma já considerada como retalho) são contabilizadas pelo modelo como perdas na função objetivo. Após a obtenção de uma solução ótima, separou-se, então, manualmente, os comprimentos considerados perdas e os comprimentos que teriam valor suficiente para serem guardados para posterior utilização, e recalculou-se o novo valor de perda, determinando-se o real número de retalhos obtidos pela solução.

Na segunda e terceira colunas da Tabela 2, estão apresentados, respectivamente, o número total de itens e de tipos de itens em cada um dos 43 exemplos da Tabela 1. Os tempos de execução dos modelos 1 e 2 requerido pelo GAMS/CPLEX em cada exemplo são apresentados na quarta e quinta colunas (em segundos). Note que, em geral, o modelo 2 demanda um tempo de execução bem menor do que o modelo 1 , possivelmente por envolver um número menor de variáveis e restrições (no exemplo 3 das tabelas, o modelo 1 possui 94 variáveis e 140 restrições, enquanto o modelo 2 possui 65 variáveis e 31 restrições). Nos exemplos nos quais os modelos não obtiveram uma solução ótima, o limite do tempo de execução aparece na Tabela 2 assinalado com um asterisco. O modelo 2 foi, em média, 49,5\% (96,35 vs. 48,63) mais rápido do que o modelo 1, considerando apenas os exemplos que obtiveram uma solução ótima dentro de limite de tempo estipulado. $\mathrm{O}$ modelo 1 foi mais rápido que o modelo 2 em apenas 7 dos 50 exemplos (exemplos 10, 15, 22, 27, $28,31$ e 37$)$.

\section{Experimento 2}

Neste experimento, comparamos as soluções obtidas pelo modelo 2 (com o GAMS/CPLEX) com as obtidas pela heurística Residual por Arredondamento Guloso (versão 2 - RAG R2), proposta recentemente por Cherri (2006) e Cherri et al. (2008), e que é um exemplo da abordagem c) mencionada na Seção 3. Essa heurística residual de arredondamento guloso utiliza a heurística construtiva $\mathrm{FFD}_{\mathrm{r}}$ ( first fit decreasing with parameter $\mathrm{r}$ ) para resolver o problema residual que é gerado por padrões de corte que são formados ao final da análise de todos os padrões que foram gerados e tem também incorporada na sua concepção a geração de retalhos para evitar perdas. Em oposição às heurísticas construtivas que geram um bom padrão de corte e o utilizam à exaustão, as heurísticas residuais consistem basicamente em resolver o problema relaxado e, em seguida, obter uma solução inteira apro- 
Tabela 2. Resultados do Experimento 1, tempo de execução dos exemplos da Tabela 1 (em segundos).

\begin{tabular}{|c|c|c|c|c|}
\hline Ex. & $\begin{array}{l}\text { Total de } \\
\text { itens }\end{array}$ & $\begin{array}{c}\text { Tipos } \\
\text { de itens }\end{array}$ & Modelo 1 (s) & Modelo 2 (s) \\
\hline 1 & 7 & 4 & 0,44 & 0,14 \\
\hline 2 & 14 & 4 & 12,01 & 0,25 \\
\hline 3 & 21 & 4 & 433,29 & 33,48 \\
\hline 4 & 10 & 4 & 1,03 & 0,59 \\
\hline 5 & 15 & 4 & 25,23 & 15,32 \\
\hline 6 & 20 & 4 & 835,88 & 24,37 \\
\hline 7 & 36 & 20 & $* 3600,04$ & 1009,52 \\
\hline 8 & 13 & 7 & 0,83 & 0,53 \\
\hline 9 & 26 & 7 & 35,95 & 5,22 \\
\hline 10 & 16 & 5 & 0,08 & 0,10 \\
\hline 11 & 24 & 5 & 0,14 & 0,11 \\
\hline 12 & 32 & 5 & 0,91 & 0,15 \\
\hline 13 & 40 & 5 & 9,22 & 0,26 \\
\hline 14 & 62 & 30 & $* 3600,03$ & 285,28 \\
\hline 15 & 8 & 2 & 0,14 & 0,17 \\
\hline 16 & 12 & 2 & 1,18 & 0,62 \\
\hline 17 & 16 & 2 & 4,87 & 1,89 \\
\hline 18 & 20 & 2 & 13,03 & 10,07 \\
\hline 19 & 29 & 17 & $* 3600,03$ & 5,59 \\
\hline 20 & 13 & 7 & 47,71 & 10,81 \\
\hline 21 & 26 & 7 & $* 3600,03$ & 0,22 \\
\hline 22 & 10 & 6 & 0,11 & 0,21 \\
\hline 23 & 20 & 6 & 550,61 & 31,39 \\
\hline 24 & 20 & 4 & 4,59 & 2,40 \\
\hline 25 & 24 & 4 & 6,65 & 4,76 \\
\hline 26 & 28 & 4 & 50,81 & 37,91 \\
\hline 27 & 10 & 5 & 0,15 & 0,18 \\
\hline 28 & 20 & 5 & 0,10 & 0,11 \\
\hline 29 & 58 & 33 & 85,41 & 55,99 \\
\hline 30 & 40 & 27 & 8,32 & 2,47 \\
\hline 31 & 80 & 27 & 1,52 & 84,90 \\
\hline 32 & 9 & 5 & 0,15 & 0,10 \\
\hline 33 & 18 & 5 & 4,10 & 0,82 \\
\hline 34 & 8 & 4 & 0,25 & 0,15 \\
\hline 35 & 16 & 4 & 5,85 & 2,29 \\
\hline 36 & 14 & 4 & 95,42 & 24,06 \\
\hline 37 & 8 & 5 & 0,10 & 0,12 \\
\hline 38 & 16 & 5 & 1322,15 & 14,65 \\
\hline 39 & 24 & 5 & $* 3600,03$ & 394,06 \\
\hline 40 & 10 & 3 & 0,17 & 0,14 \\
\hline 41 & 15 & 3 & 1,23 & 0,65 \\
\hline 42 & 20 & 3 & 15,56 & 6,47 \\
\hline 43 & 25 & 3 & 243,24 & 75,87 \\
\hline 44 & 32 & 5 & 47,84 & 23,04 \\
\hline 45 & 16 & 4 & 11,16 & 2,04 \\
\hline 46 & 100 & 4 & 254,15 & 198,74 \\
\hline 47 & 120 & 6 & 61,25 & 0,18 \\
\hline 48 & 39 & 7 & 0,22 & 0,01 \\
\hline
\end{tabular}

** Considerando apenas os exemplos com soluções com certificado de otimalidade dentro do tempo estipulado.
Tabela 2. Continuação...

\begin{tabular}{ccccc}
\hline Ex. & $\begin{array}{c}\text { Total de } \\
\text { itens }\end{array}$ & $\begin{array}{c}\text { Tipos de } \\
\text { itens }\end{array}$ & Modelo 1 (s) & Modelo 2 (s) \\
\hline 49 & 62 & 34 & $* 3600,00$ & $* 3600,00$ \\
50 & 20 & 6 & 46,17 & 14,34 \\
Média & 26,84 & 7,66 & 516,78 & 119,65 \\
& & & $(* * 96,35)$ & $(* * 48,63)$ \\
\hline
\end{tabular}

** Considerando apenas os exemplos com soluções com certificado de otimalidade dentro do tempo estipulado.

ximada, repetindo esses dois passos até a convergência da solução para a demanda requisitada. Entre as heurísticas estudadas em Cherri (2006) e Cherri et al. (2008), a heurística RAG R2 foi a que melhor se comportou em exemplos com características similares às do presente estudo, com poucos itens demandados e baixa repetição de alguns desses itens. Esta heurística também teve um bom desempenho quando comparada às heurísticas propostas em Gradisar et al. (1997, 1999a, 1999b, 2002).

Foram selecionados aleatoriamente 13 exemplos da carteira de pedidos da aeronave Ipanema, fornecida pela empresa Neiva, respectivamente, os exemplos 7, 19, 27, $29,30,32,44,45,46,47,48,49$ e 50 do experimento 1. Nesses exemplos, a quantidade total de itens varia entre 9 e 120 itens, e a quantidade de tipos de itens (m) varia entre 4 e 34 tipos de itens. Conforme o experimento 1, os comprimentos padrões dos objetos em estoque podem ser de três tamanhos diferentes: $6.000,3.500$ ou $3.000 \mathrm{~mm}$, e cada exemplo utiliza apenas um destes comprimentos.

Os resultados do experimento 2 são apresentados nas Tabelas 3 e 4 . As três primeiras colunas referem-se aos resultados do modelo 2. Cada coluna corresponde a um limite diferente do número máximo de retalhos na Equação 6 (1, 2 e 3 retalhos, respectivamente). A última coluna da Tabela 3 refere-se aos resultados obtidos pela heurística. As perdas calculadas são mostradas na Tabela 3. Em todos os exemplos, o modelo 2 obteve uma solução igual ou melhor do que a heurística, em termos de perda, e com um número igual ou menor de retalhos, dentro do limite de tempo computacional. Na última linha da tabela, estão calculadas as perdas médias de cada coluna. Na Tabela 3, encontra-se entre parênteses o número de objetos utilizados para resolver cada exemplo, e o número de retalhos obtido, também entre parênteses. Nos exemplos 3, 8 e 11, as soluções do modelo 2 (dentro do limite de tempo estipulado) são melhores do que as soluções da heurística do ponto de vista da perda percentual, considerando o mesmo número de retalhos (último número entre parênteses em cada célula da tabela).

A Tabela 4 mostra os tempos de execução dos exemplos, que são aceitáveis para a prática da empresa, considerando os ganhos com a solução e o tempo de execução do plano de corte determinado. A heurística é em geral mais rápida, com um tempo médio de 82 segundos, enquanto o 
Tabela 3. Resultados do Experimento 2, perdas (em porcentagem), número de objetos utilizados (entre parênteses) e número de retalhos gerados (entre parênteses).

\begin{tabular}{ccccc}
\hline & & & & \\
Mx. & $\sum_{j=1}^{n} u_{j} \leq 1$ & $\sum_{j=1}^{n} u_{j} \leq 2$ & \multirow{2}{*}{$\sum_{j=1}^{n} u_{j} \leq 3$} & \\
\hline 1 & $0,00 \%(4)(1)$ & $0,00 \%(4)(2)$ & $0,00 \%(4)(2)$ & $0,00 \%(4)(1)$ \\
2 & $0,00 \%(6)(1)$ & $0,00 \%(6)(1)$ & $0,00 \%(6)(1)$ & $0,00 \%(6)(1)$ \\
3 & $0,13 \%(2)(1)$ & $0,00 \%(2)(2)$ & $0,00 \%(2)(2)$ & $0,41 \%(2)(1)$ \\
4 & $0,10 \%(4)(1)$ & $0,00 \%(4)(2)$ & $0,00 \%(4)(2)$ & $0,00 \%(4)(2)$ \\
5 & $0,00 \%(3)(1)$ & $0,00 \%(3)(2)$ & $0,00 \%(3)(2)$ & $0,00 \%(3)(1)$ \\
6 & $0,00 \%(2)(1)$ & $0,00 \%(2)(2)$ & $0,00 \%(2)(2)$ & $0,00 \%(2)(2)$ \\
7 & $2,00 \%(4)(1)$ & $0,00 \%(4)(2)$ & $0,00 \%(4)(3)$ & $0,00 \%(4)(2)$ \\
8 & $0,69 \%(3)(1)$ & $0,10 \%(3)(2)$ & $0,00 \%(3)(3)$ & $0,26 \%(3)(2)$ \\
9 & $1,39 \%(5)(1)$ & $0,39 \%(5)(2)$ & $0,00 \%(5)(3)$ & $0,39 \%(5)(2)$ \\
10 & $0,00 \%(4)(1)$ & $0,00 \%(4)(2)$ & $0,00 \%(4)(2)$ & $0,00 \%(4)(1)$ \\
11 & $0,37 \%(4)(1)$ & $0,00 \%(4)(2)$ & $0,00 \%(4)(2)$ & $0,01 \%(4)(2)$ \\
12 & $* 0,16 \%(6)(1)$ & $0,03 \%(6)(2)$ & $0,00 \%(6)(3)$ & $0,16 \%(6)(1)$ \\
13 & $2,87 \%(6)(1)$ & $1,13 \%(6)(2)$ & $0,15 \%(6)(3)$ & $1,13 \%(6)(2)$ \\
Média & $0,63 \%(4,08)(1,00)$ & $0,13 \%(4,08)(1,92)$ & $0,01 \%(4,08)(2,31)$ & $0,18 \%(4,08)(1,54)$ \\
\hline
\end{tabular}

* Esta solução não obteve certificado de otimalidade dentro do limite de tempo estipulado.

Tabela 4. Resultados do Experimento 2, tempo de execução dos exemplos (em segundos).

\begin{tabular}{|c|c|c|c|c|}
\hline \multicolumn{4}{|c|}{ Modelo 2} & \multirow[t]{2}{*}{ RAG R2 } \\
\hline Ex. & $\sum_{j=1}^{n} u_{j} \leq 1$ & $\sum_{j=1}^{n} u_{j} \leq 2$ & $\sum_{j=1}^{n} u_{j} \leq 3$ & \\
\hline 1 & 1009,52 & 17,92 & 0,30 & 76,79 \\
\hline 2 & 5,59 & 141,73 & 0,10 & 0,99 \\
\hline 3 & 0,18 & 0,12 & 0,12 & 0,11 \\
\hline 4 & 55,99 & 5,39 & 6,49 & 14,91 \\
\hline 5 & 2,47 & 0,86 & 0,03 & 0,09 \\
\hline 6 & 0,10 & 0,14 & 0,14 & 5,03 \\
\hline 7 & 23,04 & 0,32 & 0,10 & 0,22 \\
\hline 8 & 2,04 & 23,17 & 0,05 & 0,14 \\
\hline 9 & 198,74 & 520,91 & 18,04 & 122,36 \\
\hline 10 & 0,18 & 0,20 & 0,10 & 0,09 \\
\hline 11 & 0,01 & 13,16 & 15,10 & 0,27 \\
\hline 12 & $* 3600,04$ & 492,16 & 3600,01 & 847,69 \\
\hline 13 & 14,34 & 32,32 & 18,13 & 0,47 \\
\hline Média & 377,86 & 96,03 & 281,44 & 82,24 \\
\hline
\end{tabular}

* Não obteve certificado de otimalidade dentro do limite de tempo estipulado.

menor tempo médio obtido pelo modelo 2 é 378, 96 e 281 segundos, respectivamente, para os casos com limites de 1,2 e 3 retalhos.

Para mais detalhes dos experimentos 1 e 2, o leitor pode consultar Abuabara (2006).

\section{Conclusões}

O objetivo deste trabalho foi estudar o problema de corte de estoque unidimensional de tubos estruturais metálicos que aparece na fabricação de aeronave leves agrícolas (Ipanema) em uma empresa aeronáutica
(Neiva/Embraer). Foram propostos dois modelos de PIM (modelos 1 e 2) com o objetivo de minimizar as perdas do material cortado no processo de corte da empresa, considerando a possibilidade de se gerar retalhos. Os modelos foram resolvidos por meio da linguagem de modelagem GAMS usando o software de otimização CPLEX. Para a validação dos modelos, experimentos computacionais foram realizados com dados reais da empresa, e os resultados obtidos foram comparados com as soluções obtidas por uma heurística construtiva da literatura e com as soluções utilizadas pela programação manual da empresa.

Estes resultados obtidos mostraram o bom desempenho dos modelos 1 e 2 em relação à heurística e à progra- 
mação da empresa, mas requerem o uso de um pacote comercial de otimização. Cerca de $65 \%$ das soluções obtidas para os modelos são melhores do que as soluções da programação da empresa, gerando aproximadamente $31 \%$ menos retalhos e utilizando $3 \%$ menos de matériaprima, o que é significativo na prática. O modelo 2 teve um desempenho superior ao modelo 1 , tanto em termos de qualidade da solução quanto de tempo computacional para obtê-la. Ele em geral obteve uma solução ótima para cada um dos exemplos analisados, dentro de um limite de tempo computacional considerado aceitável para as decisões envolvidas.

Uma pesquisa futura importante seria estudar melhor o comprimento $\mathrm{N}$ que define um retalho, considerando o melhor custo-benefício entre administrar esses objetos-retalhos e seu real valor para reaproveitamento no processo de corte da empresa. Note que o comprimento $\mathrm{N}$ é um parâmetro do modelo, que foi aqui simplesmente definido como sendo o menor comprimento de um item na carteira de pedidos. Esse valor é o praticado atualmente no processo de corte da empresa. Outros tópicos inte- ressantes seriam investigar o desempenho dos modelos 1 e 2 com as funções objetivos $\mathrm{FO}_{3}$ e $\mathrm{FO}_{4}$ definidas no final da Seção 4, que também consideram os critérios de minimizar o comprimento total cortado de objetos e minimizar o número total de retalhos gerados.

Se a empresa aumentar a taxa de produção de aviões, uma extensão interessante desta pesquisa seria estudar o trade-off entre a produtividade do equipamento de corte e o material descartado nesse processo. A empresa poderia obter maior velocidade na produção (corte) dos itens caso os padrões de corte fossem mais simples de serem executados, sob pena das quantidades de perda e retalhos serem maiores. Outra extensão interessante desta pesquisa seria estender os modelos para considerar o planejamento do corte em múltiplos períodos de produção. Neste caso, os modelos levariam em conta os benefícios de se adiantar o corte de itens e armazená-los, ao invés de gerar e armazenar objetos-retalhos, para atender às demandas dos próximos períodos. Este estudo encontra-se em andamento e seus resultados deverão ser compilados e reportados em breve.

\title{
Mixed integer programming models for the planning of one-dimensional metallic pipe cutting in the aeronautics industry
}

\begin{abstract}
This study aims to optimize the one-dimensional cutting process planning of structural metallic tubes used to build agricultural light aircrafts. Two mixed integer linear programming models are presented to minimize the waste of material cut and considering the possibility of generating surpluses with sizes sufficiently large for reuse (leftovers). The models are solved using a commercial modeling language and an optimization solver. For the validation of the models, two computational experiments were performed with actual data from the portfolio of a light aircraft designed for agricultural purposes, the Ipanema, produced by the Brazilian company Neiva/Embraer. The solutions of the models are compared with the solutions of a constructive heuristic of the literature and the solutions used by the company. The results show that the models are useful for being used in the planning of this cutting process.
\end{abstract}

Keywords: Planning of pipe cutting process. Mixed integer programming. Aeronautics industry. One-dimensional cutting and packing problems.

\section{Referência bibliográficas}

ABUABARA, A. Otimização no corte de tubos estruturais: aplicação na indústria aeronáutica agrícola. São Carlos, 2006. Dissertação - (Mestrado em Engenharia de Produção), Departamento de Engenharia de Produção, Universidade Federal de São Carlos - UFSCar.

ALVIM, A. C. F.; RIBEIRO, C. C.; GLOVER, F.; ALOISE, D. J.

A hybrid improvement heuristic for the one-dimensional bin packing problem. Journal of Heuristics, v. 10, n. 2, p. 205-229, 2004.

ARENALES, M. N.; MORABITO R.; YANASSE, H. H. Cutting and packing problems. Pesquisa Operacional, v. 19, n. 2, p. 107-299, 1999.

ARMBRUSTER, M. A solution procedure for a pattern sequencing problem as part of a one-dimensional cutting stock problem 
in the steel industry. European Journal of Operational Research, v. 141, n. 2, p. 328-340, 2002.

BROOKE, A.; KENDRIK, D.; MEERAUS A.; ROSENTHAL R. GAMS: A User's Guide. Washington: GAMS Development Co, 1998.

CHERRI, A. C. O problema de corte de estoque com reaproveitamento das sobras de material. São Carlos, 2006. Dissertação - (Mestrado em Ciências da Computação e Matemática Aplicada), Instituto de Ciências e Matemática Computacional, EESC/USP.

CHERRI, A. C.; ARENALES, M. N.; YANASSE, H. H. The one dimensional cutting stock problems with usable leftover: A heuristic approach. European Journal of Operational Research 2008 (doi10.1016/j.ejor.2008.04.039).

DYCKHOFF, H. A new linear programming approach to the cutting stock problem. Operations Research, v. 29, n. 6, p. 1092-1104, 1981.

. A Typology of Cutting and Packing Problems. European Journal of Operational Research, v. 44, n.2, p. 145-159, 1990.

DYCKHOFF, H.; ABEL, D.; GAL, T. Trim-loss and related problems. Omega, v.13, p. 59-72, 1985.

DYCKHOFF, H.; FINKE U. Cutting and Packing in Production and Distribution: Typology and Bibliography. Heidelberg: Springer-Verlag Co., 1992.

GAREY, M.; JOHNSON, D. Computers and Intractability - A Guide to the Theory of NP-Completeness. Nova York: W.H. Freeman and Co.,1979.

GILMORE, P.; GOMORY, R. A Linear Programming Approach to the Cutting Stock Problem. Operations Research, v. 9, n. 6, p. $849-859,1961$.

GILMORE, P.; GOMORY, R. A Linear Programming Approach to the Cutting Stock Problem, part II. Operations Research, v. 11, n. 6 , p. 94-120, 1963.

GRADISAR, M.; JESENKO; J.; RESINOVIC; C. Optimization of roll cutting in clothing industry. Computers \& Operational Research, v. 24, n. 10, p. 945-953, 1997.

GRADISAR, M.; KLJAJIC, M.; RESINOVIC, C.; JESENKO, J. A sequential heuristic procedure for one-dimentional cutting. European Journal of Journal of Operational Research, v. 114, n. 3, p. 557-568, 1999a.

GRADISAR, M.; RESINOVIC, C.; KLJAJIC, M. A hybrid approach for optimization of one dimentional cutting. European
Journal of Journal of Operational Research, v. 119, n. 3, p.719-728, 1999b.

Evaluation of algorithms for one-dimensional cutting. Computers \& Operations Research, v. 29, n. 9, p.1207-1220, 2002.

LASDON L. Optimization Theory for Large Systems. Nova York: Macmillan, 1970.

NEIVA - INDÚSTRIA AERONÁUTICA NEIVA LTDA. Assessoria, 2006. Disponível em: <http://www.aeroneiva.com. br>. Acesso em: 14 jun. 2006.

PILEGGI, G.; MORABITO, R.; ARENALES, M. Abordagens para otimização integrada dos problemas de geração e sequenciamento de padrões de corte: caso unidimensional. Pesquisa Operacional, v. 3, n. 25, p. 417-447, 2005.

POLDI, K. C. Algumas Extensões do Problema de Corte de Estoque. São Carlos, 2003. Dissertação - (Mestrado em Ciências da Computação e Matemática Aplicada), Instituto de Ciências e Matemática Computacional, EESC/USP.

SICUP - SPECIAL INTEREST GROUP ON CUTTING AND PACKING. Disponível em: <http://www.apdio.pt/sicup/> Acesso em: 2007.

SILVEIRA, V. R. Cenário da aviação agrícola no Brasil. São José dos Campos, 2004. Dissertação - (Mestrado em Infra-Estrutura Aeronáutica), Departamento de Engenharia de Infra-Estrutura Aeronáutica, Instituto Tecnológico de Aeronáutica - ITA.

SLACK, N.; CHAMBERS, C.; JOHNSTON, R. Administração da Produção. São Paulo: Atlas, 2002.

STADTLER, H. A one-dimensional cutting stock problem in the aluminum industry and its solution. European Journal of Operational Research, v. 44, n. 2, p. 209-223, 1990.

THURSTON, D. B. The World's Most Significant and Magnificent Aircraft: Evolution of the Modern Airplane. Warrendale, PA, USA: SAE International, 2000.

WÄSCHER, G.; HAUSSNER, H.; SCHUMANN, H. An Improved Typology of Cutting and Packing Problems. European Journal of Operational Research, v. 183, n. 3, p. 1109-1130, 2007

WILLIAMS, H. P. Model building in mathematical programming. Nova York: John Wiley \& Sons, 1978.

\section{Sobre os autores}

\section{Alexander Abuabara Reinaldo Morabito}

Departamento de Engenharia de Produção, Universidade Federal de São Carlos - UFSCar, Rodovia Washington Luiz, Km 235, CP 676, CEP13565-905, São Carlos, SP, Brasil, e-mails: abuabara@gmail.com; morabito@ufscar.br

Agradecimentos: Os autores agradecem aos três revisores anônimos pelos úteis comentários e sugestões, à Silvia Morales da Embraer pela colaboração à pesquisa e à Adriana Cherri por ter gentilmente cedido o código computacional da heurística RAG R2. Esta pesquisa contou com apoio da CAPES, CNPq e FAPESP. 